\title{
First results of a photometric and astrometric study of the globular cluster M 71 (NGC 6838)
}

\author{
M. Geffert ${ }^{1,2}$ and G. Maintz ${ }^{1}$ \\ 1 Sternwarte der Universität Bonn, Auf dem Hügel 71, D-53121 Bonn, Germany \\ 2 Observatoire de Bordeaux, URA 352 CNRS/INSU, BP. 89, F-33270 Floirac, France
}

Received September 7, 1998; accepted March 7, 2000

\begin{abstract}
We present the first results of a photometric and astrometric investigation of stars in the region of the globular cluster M 71 (NGC 6838). Using wide field CCD observations we determined $B$ and $V$ magnitudes of 4450 stars up to a limiting magnitude of $V \leq 18.5 \mathrm{mag}$. Relative proper motions were used to derive membership probabilities for stars with $R<3^{\prime}$ around the centre of M 71 . Our colour-magnitude diagram (CMD) of 320 probable cluster members reaches down to $V=18$. A well defined red giant bump - for the first time seen in M 71 - could be detected in our CMD. From isochrone fitting we find M 71 metal poorer and older than previously assumed. Four faint variable stars were confirmed to be members of M 71. Moreover, we detected 13 blue stragglers among our cluster members.
\end{abstract}

Key words: astrometry - (stars:) blue stragglers (Galaxy:) globular clusters: individual: M 71

\section{Introduction}

The similar age of the stars in globular clusters and their distribution in a small region of the sky makes globular clusters vital objects for the study of stellar evolution, especially of the late stages. However for the study of such clusters in crowded star fields at low galactic latitudes it is essential to separate the field stars from the cluster members to be able to work with a "clean" colour-magnitude diagram (CMD). Proper motions of stars in the region of globular clusters provide a unique possibility of getting membership information of the stars independently from other quantities (see e.g. references in Dauphole et al. 1996). Due to the limiting magnitude of the old plates such studies reach up to now mainly only $V=16.0$.

Send offprint requests to: M. Geffert
The globular cluster M 71 is located at low galactic latitude $\left(b=-5^{\circ}\right)$. Johnson photometry of this cluster was presented in the classical study of Arp \& Hartwick (1971), and CCD photometry by Richer \& Fahlmann (1988), Heasley \& Christian (1991) and Hodder et al. (1992). Most of the CCD studies present deep colour-magnitude diagrams reaching the main sequence far beyond the turnoff point, but give only sparse information of the subgiant branch. In the only existing modern proper motion study Cudworth (1985) combines high accuracy proper motions with photographic $B$ and $V$ photometry to present a CMD based on photometry and membership. However, as noted in the paper and as seen his Fig. 2, the data become statistical uncertain below the horizontal branch at $V=$ 14.5 and give also almost no information on the subgiant branch of M 71 .

We have performed CCD two-colour photometry of a field of about $20^{\prime} \times 20^{\prime}$ around M 71 with the exception of the stars in the very centre of the cluster. Our data are the base not only for the investigation of the CMD diagram of M 71, but also for a forthcoming study of the structure of the Milky Way at low galactic latitudes. Here we combine our photometric data of the cluster region with relative proper motions, in order to determine a CMD of M 71 covering mainly the subgiant branch.

\section{Photometric reduction}

Six CCD frames of M 71 obtained in 1995 with the $1.23 \mathrm{~m}$ telescope at Calar Alto observatory, and five CCD frames from 1993 and two first epoch plates from 1967 of the $1 \mathrm{~m}$ Cassegrain telescope at Hoher List observatory were used. In both CCD runs the Weitwinkel-Flächen Photometer and Polarimeter, WWFPP (Reif et al. 1994), camera was used. The observational data are given in Table 1. The scale of our CCD/telescope combinations is about 0 ".8/pixel. The seeing ${ }^{1}$ changed between $1.8^{\prime \prime}$ and $2.5^{\prime \prime}$.

\footnotetext{
1 The $V$ images are the poorer ones.
} 
Table 2. Coordinates and $B$ and $V$ magnitudes for stars in a field of $20^{\prime} \times 20^{\prime}$ around the globular cluster M 71

\begin{tabular}{llllllll}
\hline$\alpha_{2000}$ & $\delta_{2000}$ & $B$ & $\sigma_{B}$ & $V$ & $\sigma_{V}$ & $B-V$ & $\sigma_{B-V}$ \\
\hline 195257.584 & 184054.94 & 17.306 & 0.059 & 18.452 & 0.072 & 1.147 & 0.013 \\
195258.003 & 184010.46 & 15.962 & 0.016 & 17.302 & 0.097 & 1.341 & 0.081 \\
195258.203 & 184047.74 & 16.038 & 0.037 & 17.098 & 0.027 & 1.060 & 0.011 \\
195258.229 & 184001.65 & 14.433 & 0.012 & 15.864 & 0.030 & 1.431 & 0.019 \\
195258.372 & 183947.73 & 17.348 & 0.072 & 18.752 & 0.083 & 1.405 & 0.011 \\
. &. &. &. &. &. &. &. \\
\hline
\end{tabular}

Table 1. Observations of $1.23 \mathrm{~m}$ telescope of Calar Alto $(1.23 \mathrm{~m}$ $\mathrm{CA}$ ) and $1 \mathrm{~m}$ Cassegrain telescope of Hoher List observatory (1 $\mathrm{m}$ OHL) used in this work

\begin{tabular}{lllr}
\hline Image number & Filter & Date & Exp.Time $(\mathrm{sec})$ \\
\hline CCD 1.23 m CA: & & & \\
pf64360 & $B$ & 22101995 & 600 \\
pf64361 & $B$ & 22101995 & 1200 \\
pf64362 & $B$ & 22101995 & 600 \\
pf64440 & $V$ & 23101995 & 120 \\
pf64441 & $V$ & 23101995 & 300 \\
pf64445 & $V$ & 23101995 & 300 \\
& & & \\
CCD 1 m OHL: & & & \\
pf21623 & $V$ & 28061993 & 600 \\
pf21624 & $V$ & 28061993 & 600 \\
pf21625 & $B$ & 28061993 & 120 \\
pf21632 & $V$ & 28061993 & 300 \\
pf21633 & $R$ & 28061993 & \\
& & & \\
Plates 1 m OHL: & & & \\
C62 & $V$ & 30071967 & 5400 \\
C68 & $B$ & 28081967 & \\
\hline
\end{tabular}

For the reduction of the CCD-images we used standard CCD reduction including BIAS subtracting, flatfielding etc. and DAOPHOT under IRAF to determine positions and instrumental magnitudes of all stars on the frames. Only the CCD frames from Calar Alto were used for photometry. For the transformation of the instrumental magnitudes of our different frames to absolute magnitudes we used photometric data of 40 stars from the study of Hodder et al. (1992). A linear equation including a colour term was used in the reduction. The rms of the deviations of our data from the data of Hodder et al. (1992) amounts to $0.04 \mathrm{mag}$ in $V$ and $0.05 \mathrm{mag}$ in $B$, being smaller for the bright stars and larger for the faint ones. Having transformed the instrumental magnitudes of each frame into standard ones, we calculated the mean magnitude of each star. From the Calar Alto frames listed in Table 1 we constructed three independent CMD's using different combinations of the $B$ and $V$ observations. All these diagrams showed the same features, only the CMD using frame pf64362 was not as deep as the others. Therefore

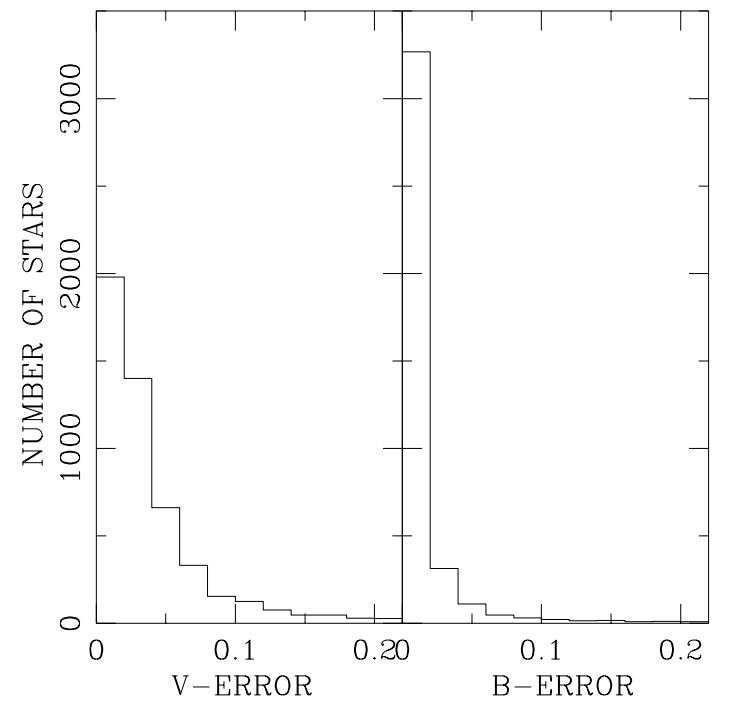

Fig. 1. The distribution of the mean errors of the mean values of $V$ and $B$

our final photometric data are based on the frame combinations pf64360/pf64441 and pf64361/pf64440. Figure 1 shows the distribution of the mean errors of mean values of the $V$ and in $B$ magnitudes for all stars, for which photometric data in $B$ and $V$ were available. It is seen, that the accuracy of the $B$ data is in general better than the accuracy of the $V$ data corresponding to the quality of the different nights, in which the observations were taken. The catalogue of our photometric data is presented in Table 2, which is completely available at the CDS in Strasbourg via anonymous ftp. As an example we give in this paper the first five lines. The errors of $B, V$ and $B-V$ in Table 2 and Fig. 1 are the rms values from the intercomparison of the two independent colour magnitude diagrams.

\section{Proper motions, separation of the cluster members, space motion}

\subsection{CCD astrometry}

CCD observations have been used in astrometry for a wide range of applications (see e.g. Monet 1994; Geffert 1998). In general for small fields an astrometric accuracy of 
0.1 pixel and better was obtained. Here we have used a wide field CCD telescope combination consisting of telescope, focal reducer and CCD chip. This equipment was tested earlier using observations of the open cluster M 67 (Geffert 1998). From a comparison frame to frame and frame to an independent catalogue we obtained an astrometric accuracy better than 50 mas for a single frame.

Combining several frames of the quoted accuracy with measurements of two photographic plates (scale: 20 ". $0 / \mathrm{mm}$, positional accuracy: $\pm 0.002 \mathrm{~mm}$ ) with an epoch difference of 30 years one would expect a proper motion accuracy better than 1.5 mas/year. Thus it seems, that a combination of observations used in this work may give proper motion results, which are of the order of the Hipparcos proper motion accuracy, but reaching magnitudes up to $V=18$ !

\subsection{Astrometric reduction, separation of cluster members}

Proper motions were used to separate members and field stars in the field of M 71. The proper motions were based on two deep first epoch plates taken in 1967 with the $(D=$ $1 \mathrm{~m}, f=12 \mathrm{~m}$ ) Cassegrain telescope of Hoher List observatory. The scale of the plates is about 20 ". $/ \mathrm{mm}$ and the limiting magnitude of these plates is 18 in $V$. The plates were digitized at the PDS 2020GM in Münster and rectangular coordinates were determined using standard routines as described e.g. in Tucholke et al. (1994). We restricted ourselves in all steps of the reduction to stars within a $3 \sigma$ level of the errors. Due to the small area covered by our data $\left(20^{\prime} \times 20^{\prime}\right)$ neither Tycho nor Hipparcos stars could be used as reference stars in the reduction of the plates and CCD frames. Instead of that, 95 reference stars from the GSC catalogue calibrated with seven Hipparcos stars were used. Using this catalogue implies that the final proper motions are only relative ones. In all steps of the reduction the scales, distortions up to third order of the coordinates, and rotations of the frames were determined simultaneously with the positions of the stars. A central plate overlap algorithm was used for the determination of the proper motions. Proper motions of 3135 stars were obtained. No systematic offset between the Hoher List and Calar Alto frames was found. The precision of the proper motion of each star was determined from the fit of mean position and proper motion to right ascension (declination) versus time. We obtained a mean error of the mean value of a single proper motion of about $1.6 \mathrm{mas} / \mathrm{yr}$ in each coordinate. In the following we will discuss only the proper motions in the region of $R<3^{\prime}$ to the cluster centre. A visual inspection of the Palomar prints and our CCD images showed that the majority of stars of M 71 is located in this region. 825 stars remained for the further investigation.

We have compared our proper motions directly with the corresponding ones of Cudworth (1985). Figure 2

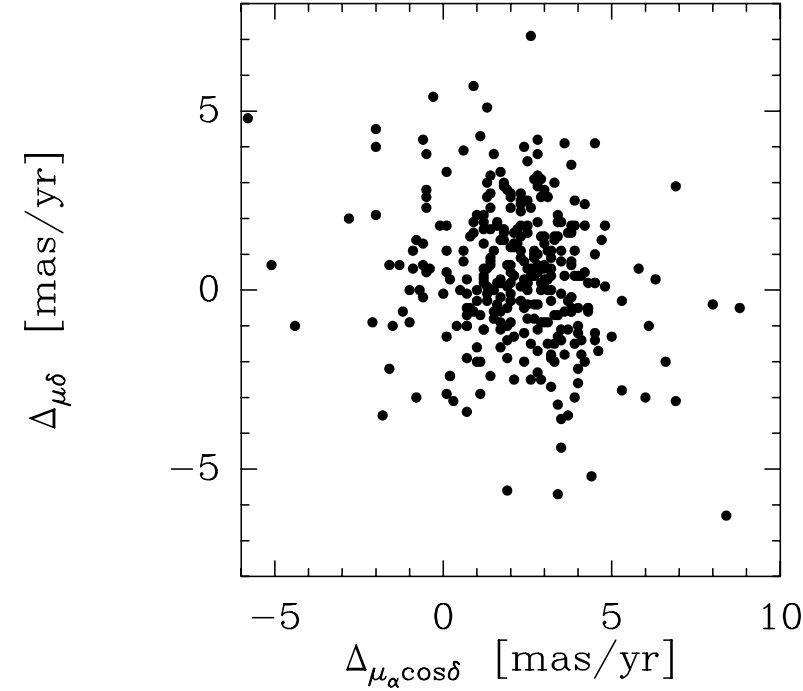

Fig. 2. Proper motion differences for 322 stars common with Cudworth. The differences are taken in the sense proper motions of Cudworth minus ours

shows the differences of the proper motions of 322 stars. However, the proper motions of the majority of our stars could not be compared with other data, since no other proper motion study of that field reaches our limiting magnitude. When comparing relative proper motions determined with respect to different reference systems, it is possible that systematic differences - especially constant offsets - exist between the proper motions of the two catalogues. Therefore only the scatter of the proper motion differences is important for the comparison of two catalogues. We found a rms of only 2 mas/yr of our differences. This is not only in good agreement with our internal errors, but points also to the fact, that the proper motion system of Cudworth differs (by chance!) not very much from ours. Membership probabilities were determined from a bivariate Gaussian fitting to the proper motions as described by Sanders (1971). All stars with proper motion components larger than 20 mas/yr were removed, since they clearly do not belong to the cluster. The parameters of the Gaussian fitting are given in Table 3, while Fig. 3 shows the distribution of the membership probability in our field. From Table 3 it is seen, that the centre of the mean cluster motion is very similar to the motion of the field stars. Therefore we expect for M 71 only a moderate separation of field and cluster stars, which is also seen in Fig. 3 by the numerous stars in the intermediate membership probability range from $10 \%$ to $60 \%$.

The cluster proper motion dispersion of about $1.9 \mathrm{mas} / \mathrm{yr}$ from Table 3 should reflect mainly the uncertainty of our proper motions and is nearly identical in both coordinates and also in good agreement with our proper motions errors. We have compared our membership designations with the corresponding ones of Cudworth and find for the stars $V<14.5$ a 


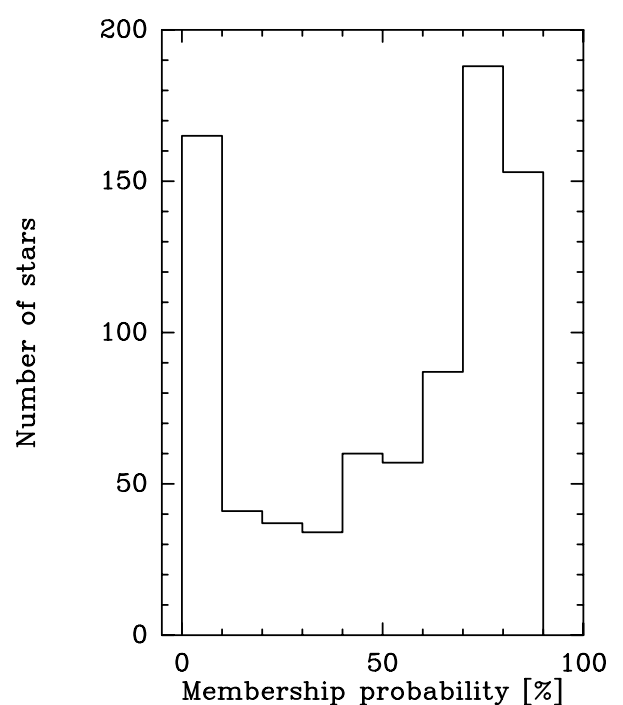

Fig. 3. The distribution of the membership probability of stars in the field of M 71

Table 3. The parameters of the bivariate Gaussian fit

\begin{tabular}{lrlr}
\hline & Value in mas/yr & & Value in mas/yr \\
\hline$\sigma_{\mathrm{c} x}$ & 1.8 & $\Sigma_{x}$ & 4.2 \\
$\sigma_{\mathrm{c} y}$ & 1.9 & $\Sigma_{y}$ & 3.9 \\
$X_{\mathrm{f}}$ & -1.5 & $X_{\mathrm{c}}$ & -2.1 \\
$Y_{\mathrm{f}}$ & -2.9 & $Y_{\mathrm{c}}$ & -1.6 \\
\hline
\end{tabular}

$\sigma_{\mathrm{c} x}, \sigma_{\mathrm{c} y}$ : Proper motion dispersion of the cluster stars. $\Sigma_{x}, \Sigma_{y}$ : Proper motion dispersion of the field stars.

$X_{\mathrm{f}}, Y_{\mathrm{f}}$ : Centre of the field star distribution.

$X_{\mathrm{c}}, Y_{\mathrm{c}}$ : Centre of the cluster star distribution.

moderate coincidence with a rms of the differences of our membership probability of $\pm 24 \%$, which increases to a rms of $\pm 36 \%$ for all stars. We will discuss the missing coincidence for the faint stars in the next chapter, when discussing the CMD.

\subsection{Absolute proper and space motion of $M 71$}

By the use of published Astrographic Catalogue positions and corresponding positions of the GSC 1.2 we have determined for 16 of our stars absolute proper motions with respect to the Hipparcos system. These stars were used to establish a preliminary link of our proper motions to the Hipparcos system. A mean cluster proper motion of $\mu_{\alpha} \cos \delta=-3.0 \pm 1.4 \mathrm{mas} / \mathrm{yr}$ and $\mu_{\delta}=-2.2 \pm 1.4 \mathrm{mas} / \mathrm{yr}$ was obtained which is in fair agreement with the $\mu_{\alpha} \cos \delta=$ $-2.3 \pm 0.5 \mathrm{mas} / \mathrm{yr}$ and $\mu_{\delta}=-5.1 \pm 0.5 \mathrm{mas} / \mathrm{yr}$ presented by Cudworth \& Hanson (1993). The larger difference in the declination components may be explained by systematic effects of the different techniques for the determination of the absolute proper motions. On the other hand due to the small distance of M 71 the consequences for the motion of the cluster within the Milky Way are neglegible: Combining our result with a distance from the sun of $3.9 \mathrm{kpc}$ of $\mathrm{M} 71$ and a heliocentric radial velocity of $v_{r}=-23.1 \mathrm{~km} \mathrm{~s}^{-1}$ (Harris 1996), we have calculated the space motion and orbit of the cluster using different galactic potentials described in Dauphole et al. (1996). As noted already in Dauphole et al. (1996) only minor differences exist between the kinematical parameters calculated with different galactic potentials. Our result supports the earlier conclusion of Cudworth \& Hanson (1993), that the cluster is on a disk-like orbit in the Galaxy. We find an orbit for M 71 with inclination of less than one degree with respect to the galactic plane with an apogalactic distance of $7.1 \pm 0.3 \mathrm{kpc}$ and a perigalactic distance of $4.9 \pm$ $0.3 \mathrm{kpc}$. We wish to point out that a more direct link of our proper motions to the Hipparcos system using Carte $\mathrm{du}$ Ciel $(\mathrm{CdC})$ plates and plates from the double refractor of Bonn is in progress. The proper motions of the complete field will be published after that link to a suited reference system.

\section{The colour-magnitude diagram of $\mathrm{M} 71$}

The colour-magnitude diagram of globular clusters at low galactic latitudes shows in principle a high degree of contamination with field stars. Additional membership information may be obtained from proper motions. However, the method of membership designation is only able to give statistical designations for each star. Figure 3 exhibits in principle three areas: mainly field stars in an membership range from $0 \%$ to $10 \%$, an intermediate range from $10 \%$ to $60 \%$ and a range of predominantly members from $60 \%$ to $90 \%$. We have set therefore the probability limit of the members to $60 \%$. Figure 4 shows the CMD for all stars with a membership probability greater than $60 \%$. The still existing field stars among our 324 probable members in Fig. 4 points to the difficulty of separating cluster and field stars with similar proper motion centres mentioned in the previous chapter. Nevertheless our CMD of M 71 shows a well populated red giant branch (RGB) from the turnoff point up to the horizontal branch. Taking the number of RGB stars per unit area, we found a much larger fraction in our sample than Hodder et al. (1992) and Cudworth (1985). This may be explained by the remark in the paper of Cudworth, that his data become less reliable for stars below the horizontal branch, which makes his cluster selection for the faint stars doubtful. Moreover, the colourmagnitude diagram of Hodder et al. (1992) was based on a selection of cluster members according to the study of Cudworth (1985) and is therefore also dependent on possible systematic effects. On the other hand, our number of RGB stars per unit area is smaller than the corresponding one of Heasley \& Christian (1991). Since their field is also central but smaller than ours one would expect for centrally concentrated stars a larger number per square. 
Our data could not be compared with the data of Arp \& Hartwick (1971), because they did not include all stars of their field in the colour-magnitude diagram.

In Fig. 4 the horizontal branch is at a magnitude of $14.48 \pm 0.09$, which is in close agreement with the value of 14.44 from Hodder et al. (1992). This is not surprising, since our photometry is based on reference stars from their study. Gallart (1998) has given an overview on several structures in the CMD. In the red giant branch the evolution of the stars may be delayed by a discontinuity of the convective envelope of the star leading to a higher density of stars at the so called red giant bump (RGB) (see references in Gallart 1998). Our diagram exhibits for the first time a red giant bump (see e.g. Gallart 1998, and references herein) in M 71, which is sharply defined by 13 stars at $V=14.92 \pm 0.01$ and $B-V=1.11 \pm 0.01$.

We have also compared our Fig. 4 with the CMD of Cudworth (1985), which is the only other existing CMD based on a selection of cluster members by proper motions. The CMD of Cudworth shows the horizontal branch and the red giant branch up to $V=15$. In that range our CMD and the corresponding one of Cudworth show nearly the same features and both coincide in the same way as our membership probabilities mentioned in the last chapter. The red giant bump mentioned above is even visible in his CMD, but was not identified as a special feature, since below $V=15$ nearly no giant branch is seen in Cudworth's CMD. In that range our CMD exhibits a subgiant branch down to $V=17$. This is the same range in the CMD, where we had found major differences in the membership probabilities between our and Cudworths study. Due to the remark by Cudworth (1985), that his data below the horizontal branch become significantly less reliable, we conclude that for stars fainter than $V=15$ our study gives more reliable results.

For comparison, we give also in Fig. 4 the fiducial sequence of Hodder et al. (1992). Our giant branch is located about 0.05 mag bluer. A similar effect can be seen, when comparing our CMD with the fiducial sequence of 47 Tuc from Hesser et al. (1987), however above the horizontal branch the shift in colour exhibits even $0.1 \mathrm{mag}$. The deviation of the subgiant branch confirms an earlier suggestion of Heasley \& Christian (1991), who explained this deviation with a higher age of M 71 with respect to 47 Tuc. We have constructed a complete CMD of M 71 from our data of the "cleaned" CMD and from the data of Hodder et al. (1992) for the fainter $(V>17)$ stars. Moreover, we included our photometric data for all stars brighter than $V=13$, since according to Cudworth (1985) nearly all of them belong to the cluster. The compiled CMD of M 71 is given in Fig. 5. Isochrones from Bergbusch \& VandenBerg (1992) and Bertelli et al. (1994) were fitted to the compiled CMD. In general the differences between isochrones of the two papers are smaller than the differences between theory and observation. It is difficult to find a suitable isochrone, which fits in the same way the red giant branch, the sub- giant branch, the turn-off point and the main sequence of the compiled CMD. From the catalogue of Cayrel de Strobel et al. (1997) and literature herein we extracted a metallicity of $\mathrm{M} 71$ of $[\mathrm{Fe} / \mathrm{H}]=-0.80$. Hodder et al. (1992) have concluded that there is no age difference between 47 Tuc and M 71. However as can be seen from Fig. 5 the isochrone (thin line) of $[\mathrm{Fe} / \mathrm{H}]=-0.8$ and age $=15 \mathrm{Gyr}$ does not fit well our compiled CMD. On the contrary, our best fit was obtained by an isochrone with an age of 18 Gyr and a metallicity of $[\mathrm{Fe} / \mathrm{H}]=-1.02$, which is indicated by the stronger isochrone in Fig. 5. Even with an age uncertainty of \pm 2 Gyr this would clearly support the earlier result of Heasley \& Christian (1991). Recently new determinations of the absolute ages of globular clusters resulted in a significant younger age of the globular clusters (see e.g. Rosenberg et al. 1999 and references). However, the aim of this paper is only the determination of the relative age of M 71 with respect to other globular clusters and especially to 47 Tuc. Thus the main conclusion of our age discussion is, that $\mathrm{M} 71$ in contrary to the previous determination of Hodder et al. (1992) has a significant older age than 47 Tuc! Our result is in good agreement with Richtler et al. (1994), who found similar ages for metal rich clusters from isochrone fitting.

However, one should also bear in mind, that Hodder et al. (1992) have corrected the magnitudes of their brighter stars according to an unknown shift between brighter and fainter frames. Thus we cannot completely rule out systematic effects between the brighter and fainter areas of the compiled CMD in Fig. 5. Our data of M 71 result in a reddening of $E(B-V)=0.27 \pm 0.05$ and an apparent distance modulus of $(m-M)_{V}=13.60$ \pm 0.10 of the cluster, which is in good agreement to the data of Richer \& Fahlmann (1988).

For the selection of BSS candidates we used the isochrone from Hodder et al. (1992), which was extrapolated beyond the turn-off point. Since our CMD reaches nearly the turn-off point we were able to select on the basis of magnitudes, colours and membership probabilities blue straggler stars (BSS) in M 71. The independent check by proper motions is important, since the colour-magnitude diagram of the field stars shows a considerable fraction of stars in the region of the CMD of the blue stragglers. All stars in the range of \pm 1 mag from the extrapolation of the isochrone in our "cleaned" CMD presented in Fig. 4 were assumed to be BSS. We have taken into account only BSS candidates with $V$ brigther then 17.3 in order to have a clear separation to the main sequence stars at the turn-off point. The data of 13 objects are given in Table 4 . Due to the strict selection criteria, this set of blue straggler is not complete. Because of this and the incompleteness in the centre of the cluster we were not able to use our data for a statistics in order to show a possible concentration of these objects as found by Richer \& Fahlmann (1988). Unfortunately their study 


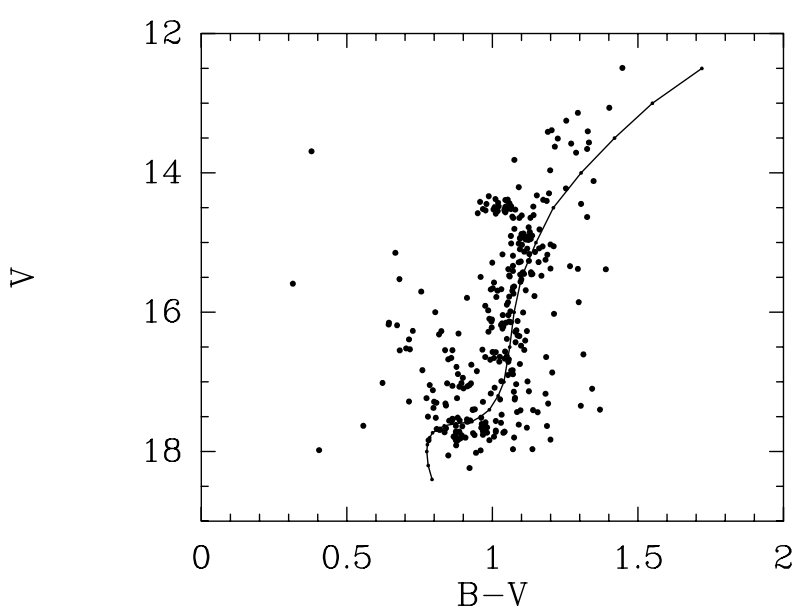

Fig. 4. The colour-magnitude diagram for the probable cluster members $(P>60 \%)$. The solid line is the fiducial sequence from Hodder et al. (1992)

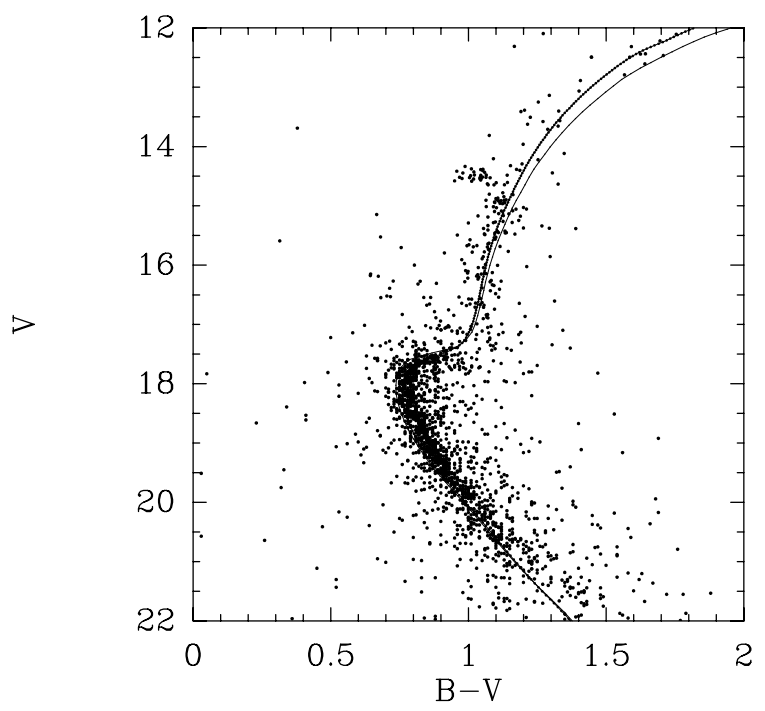

Fig. 5. Colour-magnitude diagram compiled from our probable cluster members, photometry of the bright ( $V<13$ stars), and the data of Hodder et al. (1992) for stars fainter than $V=17$. The weak isochrone corresponds to a metallicity of $[\mathrm{Fe} / \mathrm{H}]=$ -0.84 and an age of $15 \mathrm{Gyr}$, while the stronger isochrone corresponds to a metallicity of $[\mathrm{Fe} / \mathrm{H}]=-1.02$ and an age of $18 \mathrm{Gyr}$. Isochrones were taken from Bergbusch \& VandenBerg (1992)

does not give single information of their objects, which would allow a comparison with our data.

\section{Membership of variable stars}

As mentioned in the last section, our study gives for stars fainter than $V=15$ more reliable membership probabilities than the study of Cudworth (1985). Table 5 gives a list of faint variable stars found at the CDS (Strasbourg) in the region of $\mathrm{M} 71$, for which we obtained membership probabilities. Only for star H1 exists another membership probability by Cudworth (1985). According to his study
Table 4. BSS which are probable members of M 71

\begin{tabular}{lllll}
\hline Star number & $\begin{array}{l}\alpha_{2000} \\
{[\text { hhmmss.sss }]}\end{array}$ & $\begin{array}{l}\delta_{2000} \\
{[\text { ddmmss.ss }]}\end{array}$ & $V$ & $B-V$ \\
\hline 5718 & 195338.177 & 184742.77 & 17.11 & 0.82 \\
5837 & 195338.650 & 184634.43 & 17.02 & 0.82 \\
6336 & 195340.648 & 184657.48 & 17.29 & 0.83 \\
6913 & 195342.630 & 184510.87 & 16.53 & 0.66 \\
7381 & 195344.148 & 184753.09 & 16.40 & 0.73 \\
7730 & 195345.370 & 184606.76 & 15.59 & 0.32 \\
8359 & 195347.371 & 184633.02 & 17.25 & 0.79 \\
8823 & 195348.820 & 184607.79 & 16.99 & 0.67 \\
8831 & 195348.856 & 184702.55 & 16.84 & 0.71 \\
9337 & 195350.545 & 184412.87 & 17.27 & 0.81 \\
9398 & 195350.751 & 184535.33 & 17.07 & 0.81 \\
9653 & 195351.695 & 184631.96 & 16.55 & 0.70 \\
11181 & 195357.924 & 184642.79 & 17.03 & 0.83 \\
\hline
\end{tabular}

Table 5. Membership probability of some faint variable stars in M 71

\begin{tabular}{lrr}
\hline Designation & Cross Ident. & Membership Probality \\
\hline H1 & & 70 \\
H4 & YM4 & 63 \\
YM2 & & 79 \\
YM3 & & 82 \\
\hline
\end{tabular}

H Variable star from Hodder et al. (1992).

YM Variable star from Yan \& Mateo (1994).

the star is a transient case with a membership probability of $42 \%$. In contrary, Hodder et al. (1992) detected variability of this star and proposed, that H1 is a star of SX Phe type, the first of this type found in a metal rich cluster. Our study confirms this suggestion by finding a $70 \%$ membership probability. Moreover, our results indicate that the eclipsing binaries detected and studied in more detail in Hodder et al. (1992), Yan \& Mateo (1994) and McVean et al. (1997) are members of M 71.

\section{Summary}

We have presented $V$ and $B$ magnitudes of more than 4000 stars in a field of $20^{\prime} \times 20^{\prime}$. The accuracy of our data was tested by intercomparison of CMD's of different frames and by comparison with external photometry. Rms of the deviations of the comparison of our data with corresponding one of Hodder et al. (1992) were found to be 0.04 in $V$ and 0.05 in $B$.

Preliminary proper motions were obtained from two first epoch plates and recent CCD observations. The reliability of our proper motions was tested by direct comparison of the proper motions and comparison of the membership designations with the data of Cudworth (1985). We find a moderate coincidence of our results in the range 
up to $V=15$, which corresponds according to Cudworth to the range of reliability of his data. As an additional proof of our complete data ranging down to $V=18$ the cluster proper motion dispersion from the gaussian fitting of the vector-point-plot diagram is with 1.8 and $1.9 \mathrm{mas} /$ year nearly identical in both coordinates and in good agreement with our internal errors of 1.6 mas/year. A first attempt was made to link our proper motions via CdC plates and GSC data to the Hipparcos system. Our preliminary absolute proper motion led to a disk like orbit of $\mathrm{M} 71$.

Our colour-magnitude diagram shows a well established subgiant branch of M 71, which lies more bluewards than previously assumed. This shift and isochrone fitting indicate a higher age of M 71 than 47 Tuc. A red giant bump was for the first time detected in M 71. Four faint variable stars were found to be members of M 71 among them the assumed SX Phe star H1 from Hodder et al. (1992). Moreover, we found 13 blue straggler stars in the list of our cluster members.

Acknowledgements. This research has made use of the Simbad database, operated at CDS, Strasbourg, France. It is a pleasure to thank K. Cudworth for sending us his data in machine readable form. M. Geffert thanks the Observatoire de Bordeaux for kind hospitality.

\section{References}

Arp H.C., Hartwick F.D.A., 1971, ApJ 167, 499

Bergbusch P.A., VandenBerg D.A., 1992, ApJS 81, 163

Bertelli G., Bressan A., Chiosi C., Fagotto F., Nasi E., 1994, A\&AS 106, 275
Cayrel de Strobel G., Soubiran C., Friel E.D., Ralite N., Francois P., 1997, A\&AS 124, 299

Cudworth K.M., 1985, AJ 90, 65

Cudworth K.M., Hanson R.B., 1993, AJ 105, 168

Dauphole B., Geffert M., Colin J., Ducourant C., Odenkirchen M., Tucholke H.-J., 1996, A\&A 313, 119

Gallart C., 1998, ApJ 495, L43

Geffert M., 1998, CCD astrometry, in: Brosche P. et al. (eds.). The Message of the Angels - astrometry from 1798 to 1998', Proc. of the spring meeting of the Astronomische Gesellschaft, Verlag Harri Deutsch. Thun und Frankfurt am Main, p. 181-189

Harris W.E., 1996, AJ 112, 1487

Heasley J.N., Christian C.A., 1991, A differential comparison of 47 Tuc and M 71, in: Janes K. (ed.). Formation and Evolution of Star Clusters, ASP Conf. Ser. 13, San Francisco, p. 266

Hesser J.E., Harris W.E., VandenBerg D.A., Allwright J.W.B., Shott P., Stetson P.B., 1987, PASP 99, 739

Hodder P.J.C., Nemec J.M., Richer H.B., Fahlman G.G., 1992, AJ 103, 460

McVean J.R., Milone E.F., Mateo M., Yan L., 1997, ApJ 481, 782

Monet D.G., Digitization in Astronomy, in: MacGillivray H.T. et al. (eds.). Astronomy from wide-field imaging, IAU Symp. 161. Kluwer Academic Publishers, Dordrecht, p. 163

Reif K., Müller P., Leiner T., Herkendell W., Bagschik K., 1994, AG Abstr. Ser. 10, 243

Richer H.B., Fahlman G.G., 1988, ApJ 325, 218

Richtler T., Grebel E.K., Seggewiss W., 1994, A\&A 290, 412

Rosenberg A., Saviane I., Piotto G., Aparicio A., 1999, AJ 118, 2306

Sanders W.L., 1971, A\&A 14, 226

Tucholke H.-J., Brosche P., Scholz R.-D., 1994, A\&AS 104, 161

Yan L., Mateo M., 1994, AJ 108, 1810 